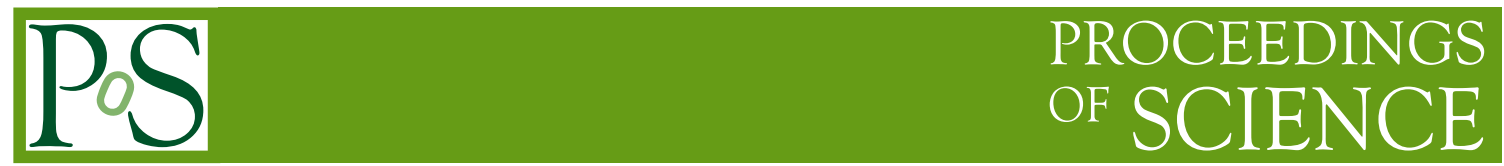

\title{
LHC Run 3 and Run 4 prospects for heavy-ion physics with LHCb
}

\section{Samuel BELIN ${ }^{a, *}$}

${ }^{a}$ Università degli studi di Cagliari, Italia

E-mail: samuel.belin@cern.ch

We report on the future upgrades of the $\mathrm{LHCb}$ detector within the scope of heavy-ion physics. A particular attention will be given to the extension of the fixed-target program as well as the performance in high-occupancy nucleus-nucleus collisions. Those technical descriptions will be followed by a discussion on the prospects for heavy-ion physics in fixed-target and collider mode.

HardProbes 2020

1-6 June 2020

Austin, Texas

${ }^{*}$ Speaker 


\section{Introduction}

Initially designed for the study of the matter-antimatter asymmetry through the measurements of b-hadrons and through the detection of rare decays, the LHCb detector [1] is a single-arm spectrometer fully instrumented at forward rapidity. Unique among the different experiments in LHC, the detector has shown great performance over the years with excellent capabilities for particle identification, vertex reconstruction and momentum measurement down to $p_{\mathrm{T}}=0$. The detector has also shown its versatility by recording not only proton-proton collisions but also $p \mathrm{~Pb}, \mathrm{PbPb}$ and fixed-target collisions, the latter enabled via injection of noble gas in the interaction region. From the physics point of view, the acceptance allows to cover a large part of the $Q^{2}-x_{\text {Bjorken }}$ phase space (see Fig. 1) and to study the largely unknown parton distribution functions of nuclei [2].

To keep up with the increasing luminosity in Run 3 (early 2022), the LHCb detector is undergoing a complete upgrade that will be presented in Sec. 2. The prospects for heavy-ion physics will be presented in Sec. 3 .
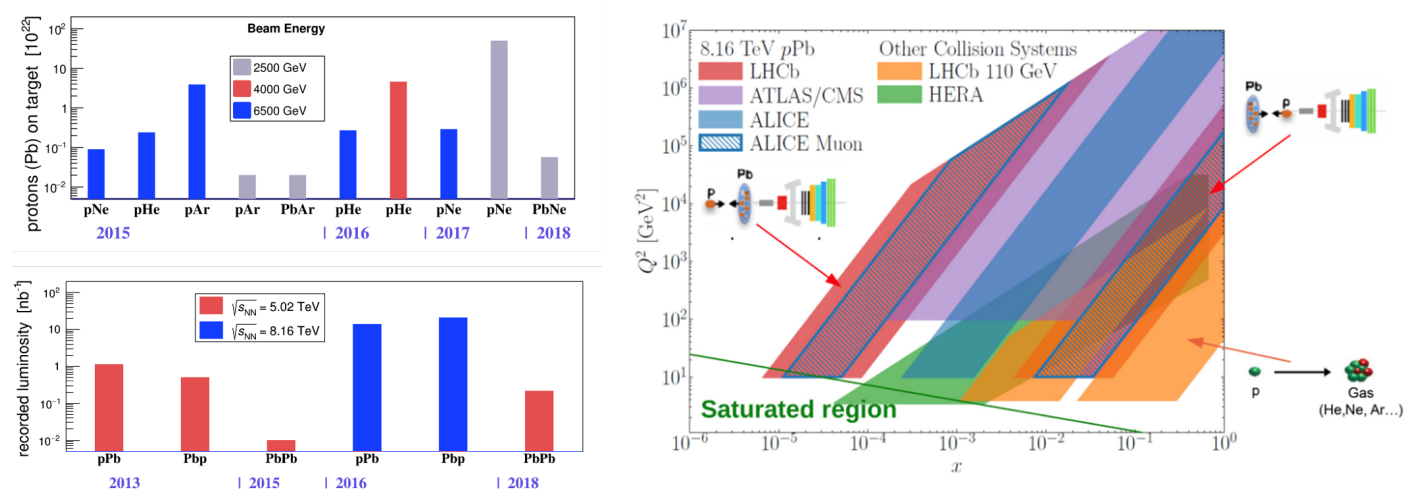

Figure 1: Left: data samples collected by LHCb in collider (top) and fixed-target (bottom) configuration. Right: $Q^{2}-x_{\text {Bjorken }}$ phase space coverage of LHCb in the different configurations and compared to other experiments.

\section{LHCb upgrades}

The LHCb collaboration is currently preparing the future of the experiment during the Long Shutdown 2 (LS2) ongoing at the LHC. A complete upgrade [3] of the LHCb detector is necessary to increase the performance for higher luminosity as well as for a higher pile-up (factor 5 increase compare to previous data taking contidtions). Thus, a new pixel VELO locator will be installed together with a new silicon micro-strip tracker called Upstream Tracker (UT). The old tracking stations ( $\mathrm{T}$ stations) are replaced by three Scintillating Fibre stations (SciFi). The optics and photodetectors of the RICH detectors will be replaced and the granularity of the muon stations increased. The calorimeter system remains almost the same with only an improvement of the readout electronics.

Finally, the new SMOG2 system [4], a standalone gas confinement tank for fixed-target measurements, will be placed upstream of the VELO system. With this new system, the gas density will be increased by a factor $\sim 100$ compared to the previous SMOG system and will provide 

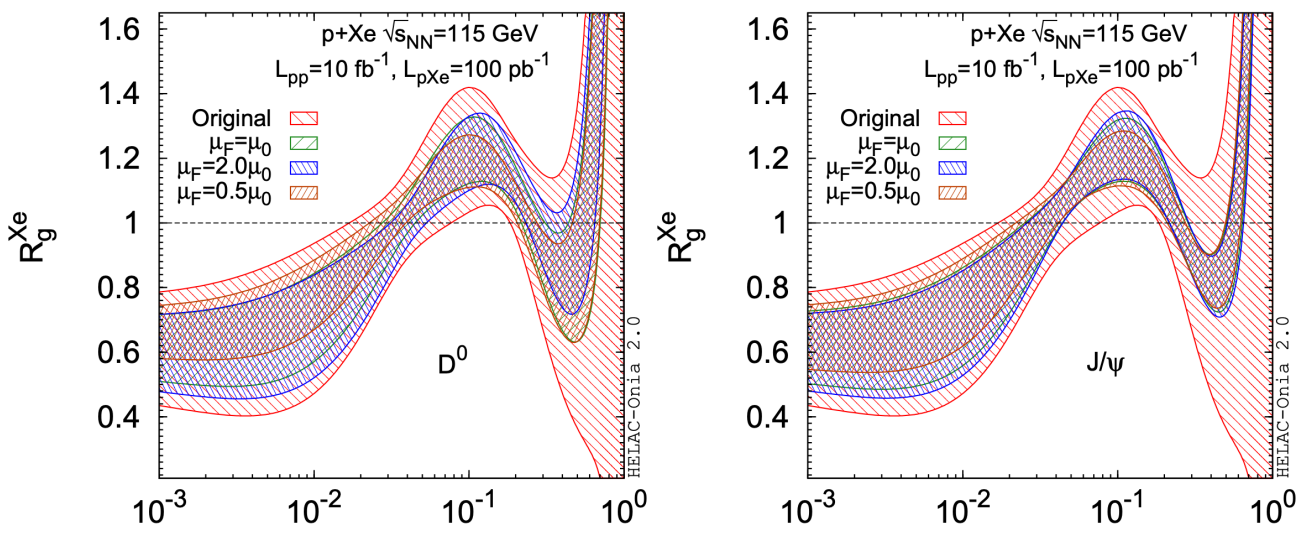

Figure 2: nCTEQ15 nPDFs before (red band) and after (green, blue and yellow band) a reweighting using results from $p$ Xe simulation at AFTER@LHCb [2].

better control on the gas injection, improving the luminosity determination. It will also be possible to inject not only noble gas but also $\mathrm{H}_{2}, \mathrm{D}_{2}$ and $\mathrm{O}_{2}$. Furthermore, undergoing studies hint on the possibility to record fixed-target collisions in parallel to pp collisions, leading to a large increase in recorded fixed-target data and thus high precision measurement.

The heavy-ion program will greatly gain from the upgrade, in particular for the performance in $\mathrm{PbPb}$ collisions, during run 2 the detector was limited to the $40 \%$ most peripheral collisions. For run 3, the first studies using simulation show no significant saturation of the detector for the 70 $\%$ most peripheral collisions, which will unlock the possibilities to perform Quark Gluon Plasma (QGP) studies during Run 3.

\section{Physics prospects}

Different predictions were made using simulated Run 3 collisions, both in collider and fixedtarget configuration. Some of them are presented in the following.

Fixed-target studies Besides the possibility to explore the phase-space diagram of the hadronic matter, as described in [2], the fixed-target data will be a solid tool to constrain nuclear parton distribution functions for large x-Bjorken and low scale $Q^{2}$, which is a crucial input for not only heavy-ion physics but also for Beyond the Standard Model and Cosmic Ray physics. Several predictions were made by the AFTER group using simulated data from an LHCb-like detector in fixed-target configuration [2]. The expected number of reconstructed events will significantly improve the precision of theoretical models of nPDFs such as nCTEQ15. Fig. 2 compares the uncertainties of the current model with a projection using pseudo-data from a fixed-target set-up in LHCb.

Collider studies The following predictions [6] are made assuming a $p \mathrm{~Pb}$ data sample at a centreof-mass energy of $\sqrt{s_{N N}}=8.8 \mathrm{TeV}$ corresponding to a total integrated luminosity of $500 \mathrm{nb}^{-1}$ which is split into two equal-sized samples of forward and backward collisions. 

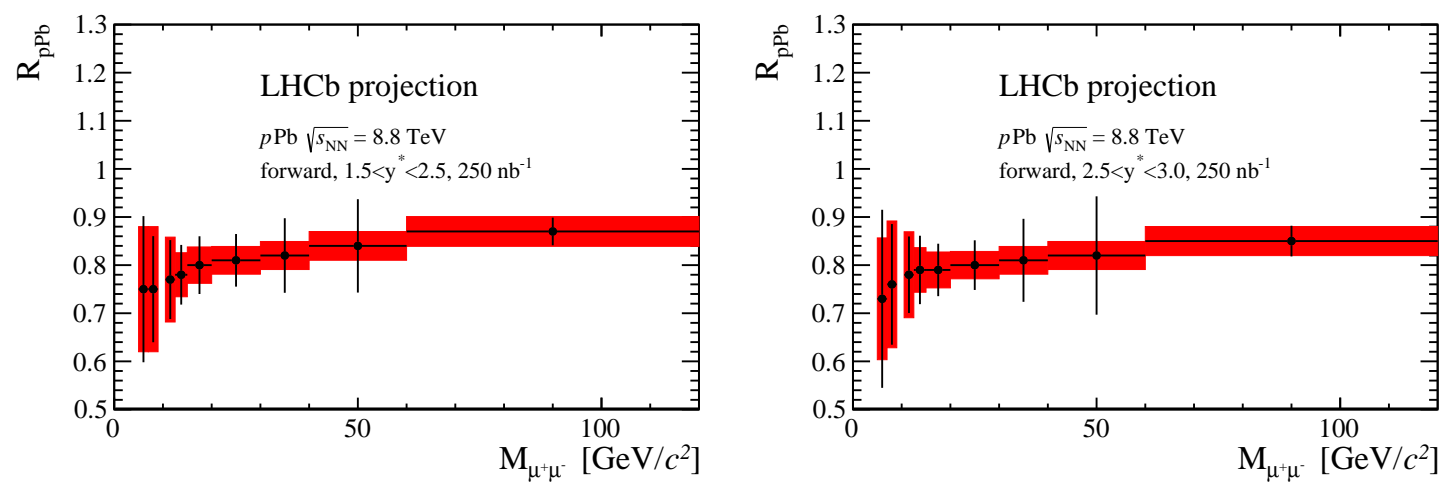

Figure 3: Projections for the Drell-Yan nuclear modification factor $\mathrm{R}_{p P b}$ as a function of the dimuon mass. The error bars represent the statistical uncertainties, while the red boxes represent the systematic uncertainties.
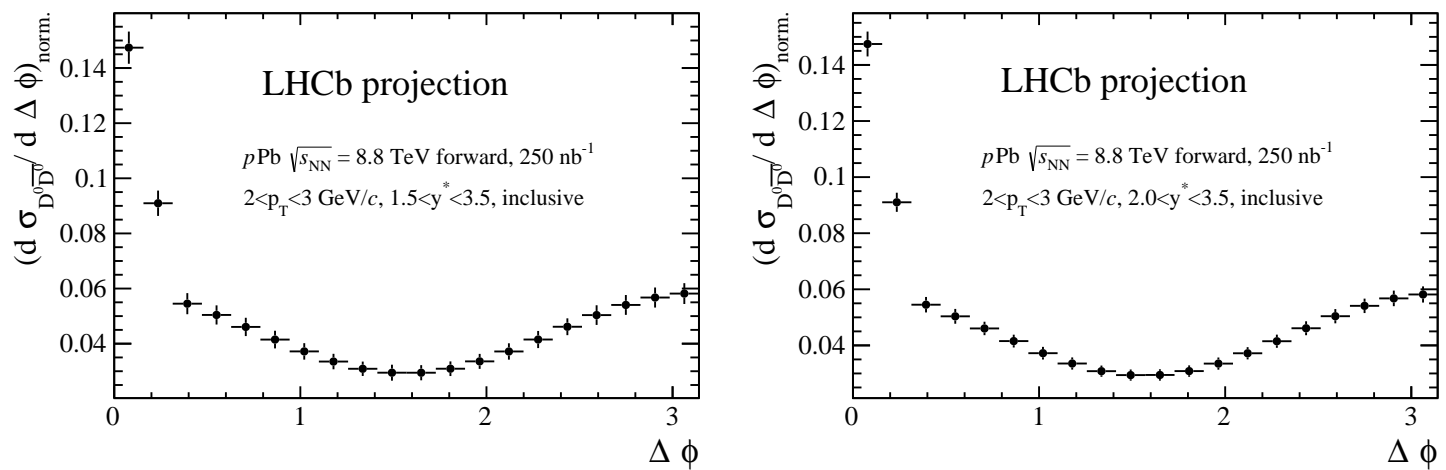

Figure 4: Projections of the normalized azimutal correlation, $\Delta \phi$, between $D^{0}$ and $\overline{D^{0}}$ in two different kinematic bin in forward $p \mathrm{~Pb}$ collision.

Drell-Yan production in $p \mathrm{~Pb}$ collisions at low dimuon mass and at forward rapidity can probe the gluon pdf at small Bjorken- $x\left(\sim 10^{-5}\right)$ where the gluon saturation could be observed. The expected good performance of the detector should allow us to perform precise studies. The Fig. 3 displays the quality of such measurement by showing the projected nuclear modification factor in $p \mathrm{~Pb}$ of the Drell-Yan process as a function of the dimuon mass in two rapidity bins.

Correlation studies between pairs of charm and beauty hadrons are of prime interest as the correlation can be linked to the saturation scale as well as taken as input to characterize the initial state with no QGP production. Projections for the azimutal correlation of $D^{0}-\overline{D^{0}}$ pairs are shown in Fig. 4. The shape of these distribution at $\Delta \phi \sim \pi$ is believed to be directly linked to the intrinsic transverse momentum of the incoming partons while at $\Delta \phi \sim 0$ the NLO process gluon splitting $(g \rightarrow c \bar{c})$ is dominant and causes the peaking structure.

Finally, precise measurements of nuclear modification factor in the beauty sector will potentially provide an answer to whether the deviation from unity is due to PDF modifications or other effects such as energy loss in a strongly interacting medium. Predictions for the $\mathrm{B}^{+}$meson production combining Run 3 and 4 are presented in Fig. 5. 

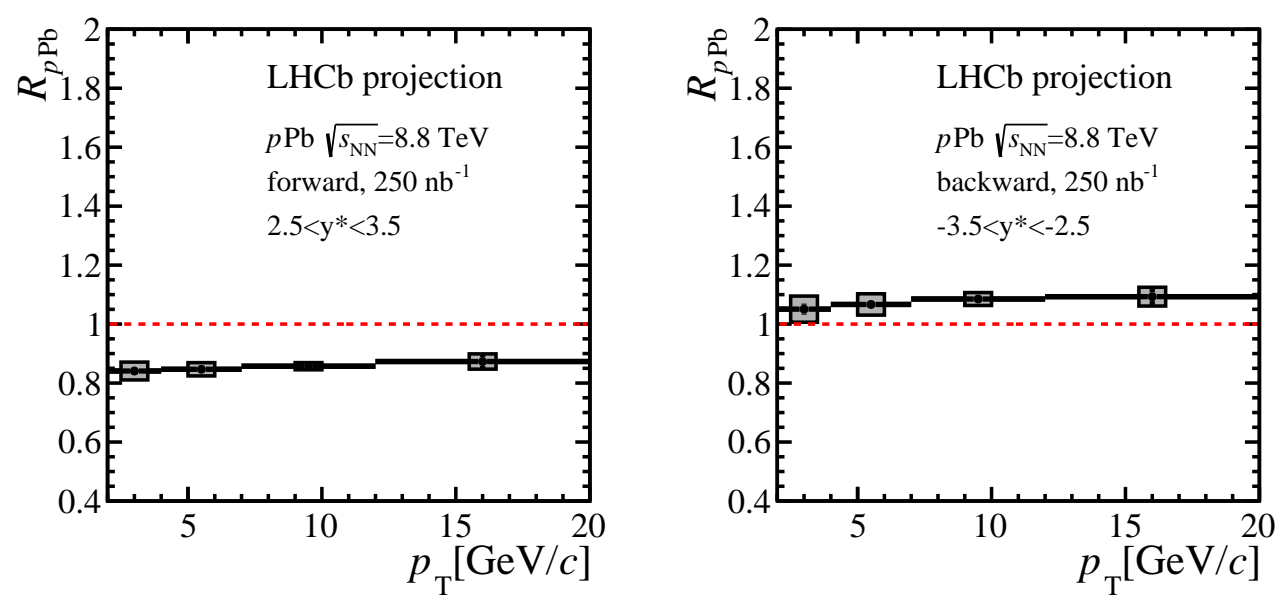

Figure 5: Projections for the nuclear modification factor $R_{p P b}$ of $\mathrm{B}^{+}$meson production in $\mathrm{LHCb}$ for Run 3 and 4.

\section{Conclusion}

LHCb has a rich heavy-ion physics program with unique capabilities. With the upcoming upgrades, the performance of the detector will be pushed one step further with, for example, the ability to reconstruct data down to $30 \%$ centrality in $\mathrm{PbPb}$ collisions during Run 3 [7]. The ambitious new fixed-target program SMOG2 will unlock unexplored energies and kinematic conditions similarly to having a completely new experiment. The current predictions in both fixed-target and collider mode show a great promise in improving the precisions of different PDFs.

\section{References}

[1] LHCb Collaboration, A.A. Alves, L.M.A. Filho, A.F. Barbosa, I. Bediaga, G. Cernicchiaro et al., The LHCb Detector at the LHC, Journal of Instrumentation 3 (2008) S08005.

[2] C. Hadjidakis, D. Kikoła, J.P. Lansberg, L. Massacrier, M.G. Echevarria, A. Kusina et al., A Fixed-Target Programme at the LHC: Physics Case and Projected Performances for Heavy-Ion, Hadron, Spin and Astroparticle Studies, arXiv:1807.00603 (2018) .

[3] A. Piucci, The LHCb Upgrade, Journal of Physics: Conference Series 878 (2017) 012012.

[4] L. Collaboration and C. (Meyrin), LHCb SMOG Upgrade, May, 2019.

[5] K. Kovař́k , A. Kusina, T. Ježo, D. Clark, C. Keppel, F. Lyonnet et al., nCTEQ15: Global analysis of nuclear parton distributions with uncertainties in the CTEQ framework, Physical Review D 93 (2016) 085037.

[6] LHCb projections for proton-lead collisions during LHC Runs 3 and 4, Nov., 2018.

[7] Upgrade simulations of VELO and SciFi saturations in PbPb collisions at $\$ \backslash$ sqrt $\left\{s_{-}\{N N\}\right\} \$=$ $5 \mathrm{TeV}$, Dec., 2019. 